\section{GW23-e0555 INHIBITION OF MICRORNA LET-7I DEPRESSES MATURATION AND FUNCTIONAL STATE OF DENDRITIC CELLS IN RESPONSE TO LPS STIMULATION VIA TARGETING SOCS1}

doi:10.1136/heartjnl-2012-302920a.31

Maomao Zhang, Jian Wu, Fang Liu, Shuo Zhang, Bo Yu. Department of Cardiology, Second Affiliated Hospital of Harbin Medical University

Objectives Dendritic cells (DCs) can initiate immune responses or confer immune tolerance depending on functional status. MicroRNAs (miRNAs) are critical for the regulation of DC function and immunity. Therefore, we try to find some miRNAs that can dynamic regulate DC maturation so as to further modulate specify antigen-specific immune responses.

Methods Bone marrow-derived DCs were generated and stimulated by LPS. To manipulate let-7i in DCs before LPS stimulation, cultured DCs were transfected with let-7i mimic or inhibitor. The maturation and functional state of DCs were assessed by Flow cytometry, MLR assay and ELISA. The subpopulation of DCs were separated by MACS. The target gene of let-7i was proved by luciferase assay, western blots, immunofluorescence and qRT-PCR.

Results We found that the miRNA let-7i was up-regulated during LPS-induced DC maturation. Down-regulation of let-7i significantly impeded DC maturation as evidenced by reduced CD80 and CD86 expression. DCs stimulated by LPS (LPS-DCs) promoted T-cell proliferation in co-culture, while LPS-stimulated DCs with down-regulated let- $7 \mathrm{i}$ were not effective at stimulating $\mathrm{T}$ cell proliferation but promoted expansion of the regulatory $\mathrm{T}$ cell (Treg) population. There were two subpopulations of LPS-stimulated DCs with down-regulated let-7i, $\mathrm{CD} 86^{-}$and $\mathrm{CD} 86^{+}$, and it was the $\mathrm{CD}^{-} 6^{-} \mathrm{DCs}$ that were more effective in inducing $\mathrm{T}$ cell hyporesponsiveness and enhancing Treg numbers, indicating that this DC population had tolerogenic properties. Furthermore, Treg with upregulated IL-10 underscored the tolerogenic effect of $\mathrm{CD}^{-} 6^{-}$DCs. Suppressor of cytokine signalling 1 (SOCS1), a crucial mediator of DC maturation, was confirmed as a let-7i target gene by luciferase construct assay. Suppression or overexpression of let-7i caused reciprocal alterations in SOCS1 protein expression, but had no significant effects on SOCS1 mRNA levels, indicating that let-7i regulated SOCS1 expression by translational suppression. The modulation of SOCS1 protein by let-7i was mainly restricted to CD86 ${ }^{-}$DCs.

Conclusions Our study demonstrates that let-7i regulation of SOCS1 is critical for LPS-induced DC maturation and immune function. Dynamic regulation of let-7i may fine tune immune responses by inducing antigen-specific immune tolerance. 